\title{
Analysis of Root Canal Anatomy and Variation in Morphology of Maxillary First Molar Using Various Methods: An In Vitro Study
}

Youssef A Algarni

\begin{abstract}
Aim: To assess and compare the internal morphology of the maxillary first molar and the incidence of the fourth root canal, especially mesiobuccal (MB2) using three diagnostic methods, clinically with or without dental operating microscopy (DOM) and cone-beam computed tomography (CBCT).

Materials and methods: A total of 336 maxillary first molar teeth were examined and distributed into the following groups: group I ( $n=112$ ), the teeth were treated by dental interns without any means of magnification. Group II $(n=112)$, the teeth were treated by an endodontist with the use of the a dental operating microscope (DOM). Group III $(n=112)$, the CBCT images were selected and examined carefully.

Results: The second mesiobuccal root canal within the maxillary first molar was present in groups I, II and III with the incidence of $27 \%, 46 \%$ and $60 \%$, respectively.

Conclusion: Within the limitations of this study, it can be concluded that the maxillary first molar teeth showed significant variations of their root canals. CBCT and dental operating microscope significantly facilitated the location and identification of the second root canal of the mesiobuccal root of maxillary first molar.

Clinical significance: Knowledge of root canal anatomy is an important factor for a successful endodontic outcome.

Keywords: Cone-beam computed tomography, Dental operating microscope, Maxillary first molar, MB2 canal.

World Journal of Dentistry (2019): 10.5005/jp-journals-10015-1644
\end{abstract}

\section{INTRODUCTION}

Since the endodontic therapy relies on the quality of a clinical procedure, knowledge of internal morphology of root canal is essential for the accomplishment of the treatment. ${ }^{1,2}$ Therefore, it is important for the clinicians to recognize common root canal morphologies and possible anatomic variations. In order to minimize the risk of treatment failure, the clinician should be aware of the chances of having extra root canals., ${ }^{3,4}$ These root canal complexities are genetically determined and hence carry importance in anthropology. ${ }^{5,6}$ The root canal anatomical variations because of differences in genetic and ethnic bases have been discussed in many studies. ${ }^{7-9}$

There are numerous studies investigating the internal morphology of the maxillary molars and primarily focusing on the presence of an extra mesiobuccal root canal in the mesial root. ${ }^{10-12}$ They have reported prevalence of the second mesiobuccal (MB2) canal of maxillary first and second molars to be quite variable. Based on the results, it has been recognized the canal incidence ranges from 3 to 4 root canals in most maxillary first molars. The reported incidence of the second canal within the mesiobuccal root has been shown to be above $50 \%$.

Different cross-sectional tomograms (CT), and lately, dental cone-beam computed tomography (CBCT) examinations, are being used in endodontics to study variation in root canal anatomy. ${ }^{13,14}$ Different methods are used to examine the morphology of roots and root canals, including tooth clearing and canal staining, ${ }^{15}$ sectioning, conventional and digital radiography, ${ }^{16}$ root canal treatment with magnification, ${ }^{17}$ modified canal staining and clearing technique, in vitro and ex vivo examination, $C T$ and $C B C T$ imagings. ${ }^{18-20}$
Department of Restorative Dental Sciences, College of Dentistry, King Khalid University, Abha, Kingdom of Saudi Arabia

Corresponding Author: Youssef A Algarni, Department of Restorative Dental Sciences, College of Dentistry, King Khalid University, Abha, Kingdom of Saudi Arabia, Phone: +966 566660604, e-mail: Yalgarni@ kku.edu.sa

How to cite this article: Algarni YA. Analysis of Root Canal Anatomy and Variation in Morphology of Maxillary First Molar Using Various Methods: An In Vitro Study. World J Dent 2019;10(4):291-294.

Source of support: Nil

Conflict of interest: None

Filho et al., in 2009, used three methods for evaluating the morphology of maxillary first molars which were clinical, ex vivo, and $\mathrm{CBCT}$. They found that CBCT imaging was a very useful diagnostic tool for identification of internal morphology of maxillary teeth as it provides a high resolution at low and effective radiation doses and is also nondestructive. It is also financially economical as compared with CT imaging and provides a three-dimensional (3D) image for assessing root canals. ${ }^{21}$

This study was conducted to compare efficacy of three methods - clinical visualization with or without a dental operating microscopy (DOM) and CBCT_for the evaluation of the internal morphology of maxillary first molars.

\section{Materials and Methods}

The present study was conducted at the College of Dentistry, King Khalid University, Abha, in collaboration with Tadawi Specialized

(c) The Author(s). 2019 Open Access This article is distributed under the terms of the Creative Commons Attribution 4.0International License (https://creativecommons org/licenses/by-nc/4.0/), which permits unrestricted use, distribution, and non-commercial reproduction in any medium, provided you give appropriate credit to the original author(s) and the source, provide a link to the Creative Commons license, and indicate if changes were made. The Creative Commons Public Domain Dedication waiver (http://creativecommons.org/publicdomain/zero/1.0/) applies to the data made available in this article, unless otherwise stated. 
Dental Center and Armed Force Hospital, Southern Region, Khamis Mushayt. A total of 336 patients, including male and female subjects from the southern region of Kingdom of Saudi Arabia, took part in the study. For conducting the study, ethical approval was procured from the ethical committee at King Khalid University, which conducts its studies as per the standards of Helsinki declaration. ${ }^{22}$ A written consent was taken from all of the patient's participating in this study. According to the method used for analysis the patients were divided into 3 groups.

\section{Group I: Canal Assessment without Magnification}

In group I (visual assessment), the records of 112 endodontic treatments of maxillary first molars performed by dental interns (under the supervision of experienced staff) during year 2018 at College of Dentistry, King Khalid University, were assessed clinically and radiographically. The number of canals and their locations and variations were evaluated.

\section{Group II: Clinical Assessment with Dental Operating Microscope}

In the clinical assessment, the records of the endodontic treatments of 112 maxillary first molars performed by an endodontist under an operating microscope during year 2018 in the Tadawi Specialized Dental Center, Khamis Mushyat, and Kingdom of Saudi Arabia were evaluated. The number of canals and their locations and variations were evaluated.

\section{Group III: Cone-beam Computed Tomographic Evaluation (Fig. 1)}

Cone-beam computed tomography images of the first maxillary molar were taken in 187 patients, which were referred to Armed Forces Hospitals Southern Region (AFHSR), Khamis Mushayt, Asir, Kingdom of Saudi Arabia. The CBCT scans were acquired from patients for different purposes which included those suffering

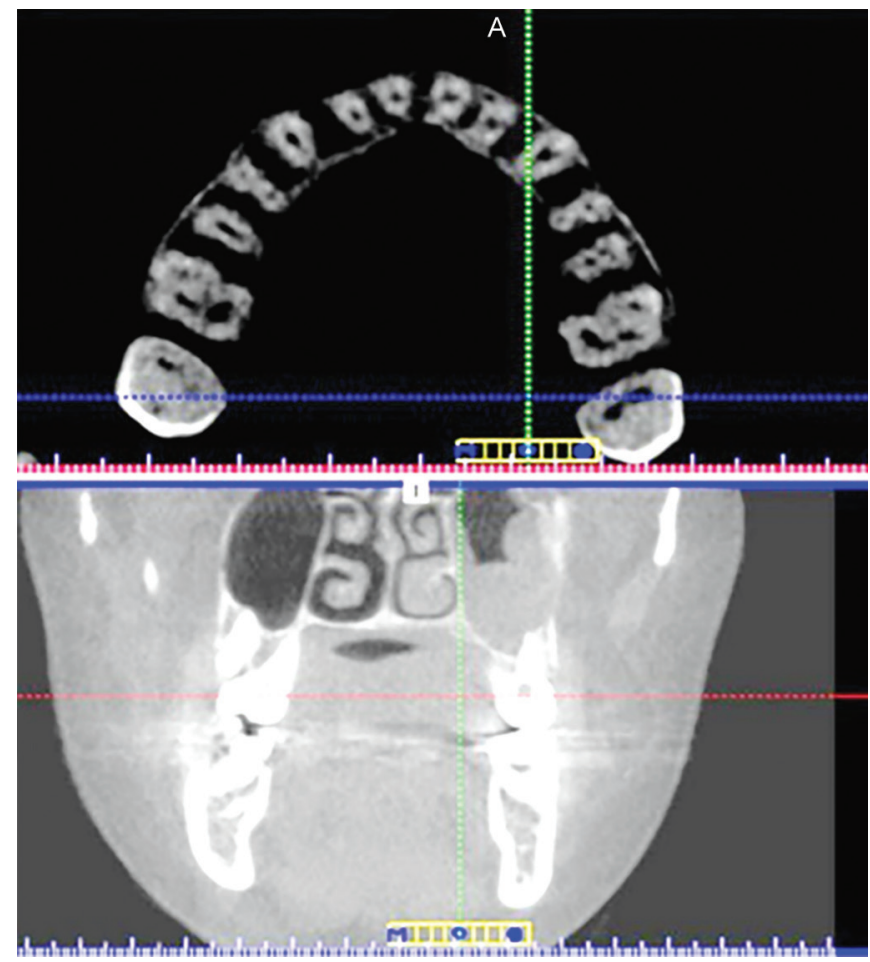

Fig. 1: CBCT image of maxillary first molar with MB2 canal from dento-alveolar trauma, tooth fracture, patients required a preoperative assessment for implants or prior to endodontic surgery, location of the impacted teeth before orthodontic treatment; and during root canal treatment on complex teeth or teeth with symptoms after root canal treatment.

Selection of teeth was done based on the following criteria:

- Maxillary permanent molar with no periapical lesions.

- No root canal filling material.

- No root canals with immature apex, resorption or calcification.

- The CBCT images of good quality.

Based on the database of patients, a total of 112 maxillary first molar teeth matched the above criteria.

The CBCT images were taken using CS 3D Imaging Software (Carestream Dental), operating at $80 \mathrm{kV}$ and $5.0 \mathrm{~mA}$, with an exposure time of 17 seconds. The voxel size and the slice thickness were $0.125 \mathrm{~mm}$ and $1.0 \mathrm{~mm}$, respectively. The field of view was $40 \times 40 \mathrm{~mm}$ or $60 \times 60 \mathrm{~mm}$ based on the examination requirements. A licensed oral radiologist performed all the CBCT scans. Evaluation of the images was done by a team that comprised experienced endodontist and radiologist. All the samples were evaluated simultaneously and separately by both the evaluators, to achieve conformity. In cases where consensus was not reached a third evaluation was done by another endodontist to reach a final consensus. Number of roots, canal configuration, the number of canals and apical foramina per root were recorded according to Vertucci's classification. ${ }^{4}$

\section{Results}

In group I (canal assessment without magnification): all 112 maxillary first molars showed $100 \%$ incidence for mesiobuccal 1 root canal, palatal root canal and distobuccal root canal. Three root canals MB1, distobuccal and palatal were found in $72.32 \%$, while four root canals MB1, MB2, distobuccal and palatal root canal were found in 27.67\% (Table 1).

In group II (clinical under DOM): all 112 maxillary first molars showed $100 \%$ incidence for mesiobuccal 1 root canal, palatal root canal and distobuccal root canal. Three root canals MB1, distobuccal and palatal were found in $53.57 \%$, while four root canals MB1, MB2, distobuccal and palatal root canal were found in $46.42 \%$.

In group III (CBCT): all 112 maxillary first molars showed 100\% incidence for mesiobuccal 1 root canal, palatal root canal and distobuccal root canal. Three root canals MB1, distobuccal and palatal were found in $39.28 \%$, while four root canals MB1, MB2, distobuccal and palatal root canal were found in $60.71 \%$ (Table 1 ).

The CBCT (group III) showed maximum incidence of mesiobuccal 2 (MB2) canal (60.71\%) followed by dental operating microscope (group II) (46.42\%) and canal assessment without magnification (group I) (27.67\%).

\section{Discussion}

The root canal anatomy requires a special attention during root canal therapy as it shows considerable variation and complexity. Proper knowledge of internal morphology of root canal is essential for optimal outcomes of the endodontic treatment. A wide range of variation has been reported in the literature with respect to the number of roots and root canals in each root in maxillary first molar.

According to Lee et al. the mesiobuccal root canal anatomy of maxillary first molars presents an endodontic challenge due to their considerable morphological variability and complexity. ${ }^{23}$ Special 
Analysis of Root Canal Morphology

Table 1: Incidence of different root canals in each root of maxillary first molar analyzed by different methods (groups)

\begin{tabular}{lllll}
\hline & $\begin{array}{l}\text { Group I (assessment without } \\
\text { magnification) }\end{array}$ & & $\begin{array}{l}\text { Group II (assessment with } \\
\text { DOM) }\end{array}$ & \\
\cline { 2 - 2 } $\begin{array}{l}\text { Number of teeth (percentage } \\
\text { of canals) }\end{array}$ & & $\begin{array}{l}\text { Number of teeth (percentage } \\
\text { of canals) }\end{array}$ & $\begin{array}{l}\text { Number of teeth (percentage of } \\
\text { canals) }\end{array}$ \\
\hline 3 root canals & $81(72.32)$ & $60(53.57)$ & $44(39.28)$ \\
4 root canals (MB2) & $31(27.67)$ & $52(46.42)$ & $68(60.71)$ \\
5 root canals & $0(0)$ & $0(0)$ & $0(0)$ \\
\hline
\end{tabular}

attention should be given during their endodontic management to prevent the undesirable consequences when they are left untreated with missed root canals. Allan et al. noticed that about $8.8 \%$ of endodontic failure cases that need retreatment were due to the presence of untreated missed canals. ${ }^{24}$

Traditionally, many methods like "champagne or bubble test" using sodium hypochlorite, staining with $1 \%$ methylene blue, canal location with sharp explorers, red line test, looking for bleeding signs, white line test and obliquely angled preoperative radiographs are used to locate extra canals. ${ }^{25}$ Technological advances have resulted in introduction of newer techniques and methods like the use of magnifying loupes, operating microscopes, computerized radiographic techniques like conventional computerized tomography, cone beam computerized tomography, X-ray computed transaxial microtomography. All these newer methods have shown to greatly enhance the negotiation of mesiobuccal root canal systems in maxillary molar. ${ }^{26,27}$

Hence this study was conducted to analyze variation in root canal anatomy, particularly in relation to mesiobuccal canal in maxillary first molar using three different methods. The first method used to locate the MB2 in the present study was with naked eye (unaided vision) and the second was the use of dental operating microscope. Introduction of microscopes to endodontics is recent, even though they have been used in medical field since decades. Reasons for introducing microscopes to endodontics include enhanced visibility and lighting. According to Stropko, ${ }^{27}$ identification of MB2 canal in maxillary first molar is enhanced clinically from 20 to $92 \%$ with the use of an operating microscope. Thus operating microscopes not only provide a great amount of light to illuminate the pulp chamber, but also magnify the chamber anatomy in great detail.

The result of group I showed that 31 out of 112 (Table 1) teeth showed MB2 canal orifice when viewed with unaided vision While the group II showed 52 out of 112 under the dental operating microscope at $20 \times$ magnification which represent $46.42 \%$. These results correlate with the study conducted by Peeters in which they conclude that of the 308 maxillary first molars studied under dental operating microscope, 211 (68.5\%) had an MB2 canal in their mesiobuccal root. ${ }^{28}$

In this study, third method used for evaluating root canal morphology was cone beam computerized tomography. Until recently, most of this core information on root canal anatomy would be obtained from conventional radiographs, but the main disadvantage with conventional film-based radiographs are the superimposition of images since they provide a 2-dimensional image of an actual 3D object. Nowadays this disadvantage is being overcome by a relatively newer diagnostic imaging modality which is cone beam computerized tomography. ${ }^{29}$

In the present study when teeth were analyzed under CBCT, 68 (60.71\%) teeth showed MB2 canal orifice (Table 1). These results were almost similar to those reported by Shenoi et al. ${ }^{29}$ who reported an incidence of $80 \% \mathrm{MB} 2$ canals under CBCT evaluation. However, Bauman in contrary to present study showed a much higher incidence of MB2 up to $92 \%$ under CBCT evaluation. ${ }^{30}$

\section{Conclusion}

According to the results of this study, it can be concluded that a preoperative $C B C T$ image and use of the dental operating microscope during endodontic treatment can significantly increase the identification of MB2 canal in a maxillary first molar.

\section{References}

1. Ashraf $\mathrm{H}$, Paymanpour $\mathrm{P}$, et al. Healing of an extensive periradicular lesion subsequent to a proper endodontic treatment of a mandibular first premolar with complex anatomy. Case Rep Dent 2013;2013:972093. DOI: 10.1155/2013/972093.

2. Chang S, Lee J, et al. In-depth morphological study of mesiobuccal root canal systems in maxillary first molars: Review. Restor Dent Endod 2013;38(1):2-10. DOI: 10.5395/rde.2013.38.1.2.

3. Weine FS, Healey HJ, et al. Canal configuration in the mesiobuccal root of the maxillary first molar and its endodontic significance. Oral Surg Oral Med Oral Pathol 1969;28:419. DOI: 10.1016/0030-4220(69) 90237-0.

4. Vertucci FJ. Root canal morphology and its relationship to endodontic procedures. Endod Top 2005;10:3-29. DOI: 10.1111/j.16011546.2005.00129.x.

5. Neelakantan P, Subbarao C, et al. Comparative evaluation of modified canal staining and clearing technique, cone-beam computed tomography, peripheral quantitative computed tomography, spiral computed tomography, and plain and contrast medium-enhanced digital radiography in studying root canal morphology. J Endod 2010;36:1547-1551. DOI: 10.1016/j.joen.2010.05.008.

6. Silva EJ, Nejaim Y, et al. Evaluation of root canal configuration of maxillary molars in a Brazilian population using cone-beam computed tomographic imaging: an in vivo study. J Endod 2014;40(2):173-176. DOI: 10.1016/j.joen.2013.10.002.

7. Chen GYH, Tong C. Investigation of the root canal configuration of mandibular first molars in a Taiwan Chinese population. Int Endod J 2009;42(11):1044-1049. DOI: 10.1111/j.1365-2591.2009.01619.x.

8. Weng XL, Yu SB, et al. Root canal morphology of permanent maxillary teeth in the Han nationality in the Chinese Guanzhong area: a new modified root canal staining technique. J Endod 2009;35:651-656. DOI: 10.1016/j.joen.2009.02.010.

9. Hosseinpour S, Kharazifard MJ, et al. Root Canal Morphology of Permanent Mandibular Premolars in Iranian Population: A Systematic Review. Iran Endod J 2016;11(3):150-156.

10. Pecora JD, Woelfel JB, et al. Morphologic study of the maxillary molars. Part II: internal anatomy. Braz Dent J 1992;3:53-57.

11. Sert S, Bayirli GS. Evaluation of the root canal configurations of the mandibular and maxillary permanent teeth by gender in the Turkish population. J Endod 2004;30:391-398. DOI: 10.1097/00004770200406000-00004.

12. Cleghorn BM, Christie WH, et al. Root and root canal morphology of the human permanent maxillary first molar: a literature review. J Endod 2006;32:813-821. DOI: 10.1016/j.joen.2006.04.014. 
13. Gahleitner A, Watzek G, et al. Dental CT: imaging technique, anatomy and pathologic conditions of the jaws. Eur Radiol 2003;13:366-376.

14. Kiarudi AH, Eghbal MJ, et al. The applications of cone-beam computed tomography in endodontics: a review of literature. Iran Endod J 2015;10(1):16-25.

15. Ng YL, Aung TH, et al. Root and canal morphology of Burmese maxillary molars. Int Endod J 2001;34:620-630. DOI: 10.1046/j.13652591.2001.00438.x.

16. Pattanshetti N, Gaidhane M, et al. Root and canal morphology of the mesiobuccal and distal roots of permanent first molars in a Kuwait population-a clinical study. Int Endod J 2008;41:755-762. DOI: 10.1111/j.1365-2591.2008.01427.x.

17. Zheng $\mathrm{QH}$, Wang $\mathrm{Y}$, et al. A cone-beam computed tomography study of maxillary first permanent molar root and canal morphology in a Chinese population. J Endod 2010;36:1480-1484. DOI: 10.1016/ j.joen.2010.06.018.

18. Neelakantan $P$, Subbarao C, et al. Cone-beam computed tomography study of root and canal morphology of maxillary first and second molars in an Indian population. J Endod 2010;36:1622-1627. DOI: 10.1016/j.joen.2010.07.006.

19. Kim Y, Lee SJ, et al. Morphology of maxillary first and second molars analyzed by cone-beam computed tomography in a Korean population: variations in the number of roots and canals and the incidence of fusion. J Endod 2012;38:1063-1068. DOI: 10.1016/j.joen.2012.04.025.

20. Rouhani A, Bagherpour A, et al. Cone-beam computed tomography evaluation of maxillary first and second molars in Iranian population: a morphological study. Iran Endod J 2014;9:190-194.

21. Baratto Filho F, Zaitter S, et al. Analysis of the internal anatomy of maxillary first molars by using different methods. J Endod 2009;35:337-342. DOI: 10.1016/j.joen.2008.11.022.
22. World Medical Association. World Medical Association Declaration of Helsinki: ethical principles for medical research involving human subjects. JAMA 2013 Nov 27;310(20):2191-2194. DOI: 10.1001/ jama.2013.281053.

23. Lee J-H. Mesiobuccal root canal anatomy of Korean maxillary first and second molars by cone-beam computed tomography. Oral Surg Oral Med Oral Pathol Oral Radiol Endod 2011;111:785-791. DOI: 10.1016/ j.tripleo.2010.11.026.

24. Abuabara A. Identification of root canals in maxillary molars using cone beam computed tomography. Rev Sul-Bras Odontol 2010;7(1):117-120.

25. Nosonowitz DM, Brenner MR. The major canals of the mesiobuccal root of the maxillary 1st and 2nd molars. NY J Dent 2003;43:12-50.

26. Neaverth EJ, Kotler LM, et al. Clinical investigation (in vivo) of endodontically treated maxillary first molars. J Endod 1997;13: 506-512. DOI: 10.1016/S0099-2399(87)80018-3.

27. Stropko JJ. Canal morphology of maxillary molars: clinical observations of canal configurations. J Endod 1999;25:446-450. DOl: 10.1016/S0099-2399(99)80276-3.

28. Peeter $\mathrm{HH}$, Suardita K, et al. Prevalence of a second canal in the mesiobuccal root of permanent maxillary first molars from an Indonesian population. J Oral Sci 2011;53:489-494. DOI: 10.2334/ josnusd.53.489.

29. Shenoi R. Endodontic applications of cone beam volumetric tomography. J Endod 2012;33:1121-1132.

30. Bauman R, Scheetz J. Ex vivo detection of mesiobuccal canals in maxillary molars using CBCT at four different isotropic voxel dimensions. Int Endod J 2011;44:752-758. DOI: 10.1111/j.13652591.2011.01882.x.World Journal of Dentistry (2019): 10.5005/ jp-journals-10015-1643 